The identity just preceding relation (5) is a generalization of that involved in the classic transformation of Abel; for, if we replace $v_{1}(x)$ by $g(x), v_{2}(x)$ by $v(x)$, and $f(x)$ by 1 , we have the Abel identity

$$
\begin{aligned}
\sum_{i=1}^{n} g\left(x_{i}\right)\left[v\left(x_{i}\right)-v\left(x_{i-1}\right)\right] & \\
& \equiv-\sum_{i=1}^{n} v\left(x_{i-1}\right)\left[g\left(x_{i}\right)-g\left(x_{i-1}\right)\right]+g(b) v(b)-g(a) v(a) .
\end{aligned}
$$

It is obvious that a repeated use of (5) reduces the integral of $f(x)$ as to a product $v_{1}(x) v_{2}(x) \cdots v_{n}(x)$ to a sum of $n$ integrals of functions as to $v_{1}(x), v_{2}(x), \cdots, v_{n}(x)$, respectively, under appropriate conditions like (6) and (7) and the hypothesis of the existence of these $n$ integrals. The question arises naturally as to whether some simple identity exists, analogous to that employed in deriving (5), which would yield the entire result at once. It was through this question that I was led to identity (1). Having it, it is natural to extend the classic theorems about convergence of series previously obtained through the particular case which yields Abel's transformation. The reader will have no difficulty in obtaining through identity (1) the transformation of a Stieltjes integral mentioned at the beginning of this paragraph.

UNIVERSITY OF ILLINOIS, August, 1919.

\title{
NOTE ON A PHYSICAL INTERPRETATION OF STIELTJES INTEGRALS.
}

BY PROFESSOR R. D. CARMICHAEL.

(Read before the American Mathematical Society September 4, 1919.)

Stifltues was led to his definition of integral by what he called the problem of moments (see $\$ 24$ of his memoir in Annales de la Faculté des Sciences de Toulouse, 1894). Consider on a straight line $O X$ a distribution of (positive) mass, the mass $m_{i}$ being concentrated at the distance $\xi_{i}$ from the origin 0 . The sum $\Sigma m_{i} \xi_{i}{ }^{k}$ he called the moment of order $k$ of the mass with respect to the origin. He aiso considered the more general distribution of mass on $O X$ which is such that 
the mass on the segment $(0 x)$ from 0 to $x$ is $\varphi(x)$ where $\varphi(x)$ is a non-decreasing function which is finite for every finite value of $x$. Let us consider the moment about $O$, of order $k$, of the mass so distributed on the interval $(a b)$ where $a$ and $b$ are both positive. Let $\pi$ be a partition of $(a b)$ of norm $\delta$ due to the points $x_{0}=a, x_{1}, \cdots, x_{n-1}, x_{n}=b$, and let $\xi_{i}$ be a point of the interval $\left(x_{i-1}, x_{i}\right)$. Then for the moment in question we should obviously have the value

$$
\lim _{\delta=0} \sum_{i=1}^{n} \xi_{i}{ }^{k}\left\{\varphi\left(x_{i}\right)-\varphi\left(x_{i-1}\right)\right\} .
$$

That is, the value of the moment of order $k$ is expressed by the Stieltjes integral

$$
\int_{a}^{b} x^{k} d \varphi(x)
$$

We thus have Stieltjes' physical interpretation of the integral (1) for each value of $k$, the funciion $\varphi(x)$ being monotonic.

The object of this note is to throw the physical interpretation of this integral into another form and to generalize the new form so as to afford a physical interpretation for the integral of $f(x)$ as to any function $v(x)$ of bounded variation.

Let us consider the curve $y=x^{k}$ and a distribution of mass on that curve such that the mass on the segment from $(0,0)$ to $(x, y)$ shall be $\varphi(x)$. Then the integral (1) represents the usual (first) moment of the mass on the segment of $y=x^{k}$ from $x=a$ to $x=b$ with respect to the axis of $x$, as one sees readily from the foregoing finite sum of which the integral (1) is the limit.

Now this interpretation of integral (1) admits of an obvious generalization. Let $f(x)$ be any single-valued function of $x$ which is bounded on $(a b)$ and let positive mass be dis'ributed along the curve $y=f(x)$ so that the mass on the segment from $x=a$ to $x=x$ shall be $\varphi(x)$, where the functions $f(x)$ and $\varphi(x)$ have no common singularity and where the total mass involved is finite. Then the integral

$$
\int_{a}^{b} f(x) d \varphi(x)
$$

exists and gives the value of the moment about the $x$-axis of the mass on the curve $y=f(x)$ from $x=a$ to $x=b$. 
Thus we have a physical interpretation for the integral of a bounded function with respect to any bounded monotonic non-decreasing function. If we avail ourselves of the notion of negative mass we may obtain similarly a physical interpretation for the integral of a bounded function $f(x)$ as to a function $v(x)$ of bounded variation.

Write $v(x)$ in the form

$$
v(x)=v(a)+P(x)-N(x),
$$

where $P(x)$ and $N(x)$ are respectively the positive and the negative variation of $v(x)$ on $(a x)$. Then along the curve $y=f(x)$ let us have a distribution of positive mass such that the positive mass on the segment from $x=a$ to $x=x$ is $P(x)$; along the same curve let us have a distribution of negative mass such that the negative mass on the segment from $x=a$ to $x=x$ is $N(x)$. We suppose that neither $P(x)$ nor $N(x)$ is discontinuous at a discontinuity of $f(x)$. Then the moment of the positive mass about the axis will be represented by the integral of $f(x)$ as to $P(x)$; that of the negative mass by the negative of the integral of $f(x)$ as to $N(x)$. Hence the algebraic sum of these moments, or the total moment about the $x$-axis, is represented by

$$
\int_{a}^{b} f(x) d v(x) .
$$

Such a physical interpretation of the integral is useful in giving one a better intuitive sense of its character and hence in affording a means of classifying its properties in his thought.

If one is disturbed by the notion of negative mass which enters into the foregoing interpretation he may avoid its use by resorting to another range of physical phenomena. He may replace positive and negative mass by positive and negative magnetism. Then $P(x)[N(x)]$ would denote the total positive [negative] magnetism on the segment of $y=f(x)$ from $x=a$ to $x=x$ and the moment would be taken with respect to the $x$-axis under a magnetic field of force of unit intensity in a direction perpendicular to the $x y$-plane. The moment will again be represented by the integral (2).

It is obvious that this representation may also be modified into a geometrical one. Consider the cylindrical surface perpendicular to the $x y$-plane and intersecting it in the curve 
$y=f(x)$. On the positive side of the $x y$-plane take the curve lying on this cylindrical surface and satisfying the condition $z=P(x)$; on the other side take the curve lying on the cylindrical surface and satisfying the condition $z=-N(x)$. We thus intercept between two curves a part of the cylindrical surface for which $x$ is on $(a b)$. It is not difficult to form a fair intuitive notion of this portion of the surface since $P(x)$ and $N(x)$ are both monotonic non-decreasing. Then the integral (2) is the "area" of the part of this bounded cylindrical surface lying on the positive side of the $x y$-plane minus the "area" of that part lying on the other side.

UNIVERSITY OF ILLINOIS, August, 1919.

\title{
A DERIVATION OF THE EQUATION OF THE NORMAL PROBABILITY CURVE.
}

\author{
BY PROFESSOR W. D. CAIRNS.
}

(Read before the American Mathematical Society September 5, 1918.)

THE symmetrical distribution of magnitudes about their mean is commonly represented by a "polygon" whose equally spaced ordinates are proportional to the terms of the expansion of $(1+1)^{n}$. The statement is frequently made in textbooks without any proof that as $n$ is increased indefinitely, the equal spaces and the vertical scale being properly controlled, the polygon approaches as its limiting form the normal curve $y=k e^{-h^{2} x^{2}}$. The method here given for the proof of this theorem consists essentially in controlling what may be called the points of inflexion of the polygon so that these points approach predetermined positions on each side of the mean. Since an extended and rigorous proof of the probability theorem has been published,* it will suffice to indicate here the general plan of the proof.

* E. L. Dodd, American Mathematical Monthly, vol. 20 (1913), p. 128.

Since this paper was written, a proof by A. A. Bennett has appeared in this Bulletin, volume 24, No. 10, page 477. In that proof the area (rather than the standard deviation, as used in the present paper) and the middle ordinate control the curve and Wallis's product formula for $\pi / 2$ is used. Since the area and the standard deviation are alike fundamental in the applications to probability, statistics, theory of errors, etc., it would seem that each of these gives a natural method of approach. 